\title{
Understanding Mobility Decision Making of People During Covid-19 with Decision Making Theory
}

\author{
Guanghan $\mathrm{Wu}^{1, *}$, Shouyan Xia ${ }^{1}$, Jiayi $\mathrm{Li}^{1}$, Hongyi Chen ${ }^{1}$, Haoxing $\mathrm{Qi}^{1}$,
} Haowen $\mathrm{Yu}^{1}$, Jouni Markkula ${ }^{2}$

\author{
${ }^{1}$ Nanjing Institute of Technology, China \\ ${ }^{2}$ University of Oulu, Finland \\ *Corresponding author. Email: guanghan.wu@outlook.com
}

\begin{abstract}
COVID-19 pandemic broke out at the beginning of 2020. Since then, it has spread all over the world. Restriction of its fatal effects demands understanding and management of human behavior, in order to mitigate the spread of the pandemic and deal with abrupt outbreaks across the globe. The objective of this study is to understand mobility decision making of people during pandemic by applying an extended Decision Field Theory (DFT) model. In the study, data about people mobility decisions was collected with a survey of three rounds that was constructed according to the structure of the extended DFT. The preliminary results of the study are presented with descriptive analysis. The results indicate that several factors affect to the decisions of mobility and they vary among the persons in time. Apart from the main factors directly affecting to the decision making, there is also indirect factors influencing to the decisions. The result of this study can be utilized for further development of a decision model for understanding and predicting decision behavior of people in pandemic situation or other corresponding conditions.
\end{abstract}

Keywords: COVID-19, Human Behavior, Mobility, Decision Making Theory.

\section{INTRODUCTION}

The whole global world, societies, economy as well as education was shaken suddenly, unexpectedly and quickly when the COVID-19 pandemic started to spread in all corners of the world. Drastic measures were taken in all countries, including governmental, business and education sectors. Innovative solutions were discovered, and quickly put in use. This is expected to leave long lasting effect, and transforms business, education and travelling.

In all the measures, the mobility of people was affected, as that is the core factor in pandemic spreading, and management. People were given regulations and restrictions related to their movements. The management of travelling varies from country to country, with varying effectiveness. People are also following the guidelines differently, depending on countries.

The novel coronavirus caused COVID-19 firstly exploded at the end of January, 2020 in Wuhan, China and shocked everyone. In order to urgently curb the rapid spread of the pandemic, all residents kept the house strictly for the Stay-At-Home order to fight against the pandemic. However, continuous outbreaks of COVID-19 rapidly occurred in the whole world.

Along the development of pandemic around the world, the situation of pandemic has been continuously changing and transforming, and big outbreaks continuously occurred across the globe. Nevertheless, huma are social and isolation can be harmful [1]. Outside and social activities are also unpreventable as the pandemic is gradually dampened in China. Over time, people kept the house for a too long period during COVID-19, becoming less afraid of COVID-19. These factors can affect their decisions about movements.

Key to be able to manage the pandemic is to understand and manage the movement of people, which is depending in large part of the decisions people make about their movements, based on their perception of the situation. A decision theory or model is needed to be applied in the study on the decision making process of movements during pandemic. 
This paper aims to contribute to this area of research by exploring the rules behind moving and travelling of people under regulations guidelines and restrictions under government. An extended decision field theory (DFT) model is involved in the process of designing and collecting relevant data, and analysing the data [2].

The overall structure of the study takes the form of 6 sections, including this introduction section. The relationship between COVID-19 and the applied DFT model is described in section 2. The development and mathematical model of DFT is introduced in section 3. Survey questionnaires were made and three rounds of online surveys were conducted according to extended DFT model, the methodology is presented in section 4. Human behavior and decision making process were analysed based on the preliminary results in section 5 . A conclusion of this research, including the limitation of this study and future work are discussed in section 6.

\section{COVID-19 AND HUMAN DECISION BEHAVIOR}

In such environment with uncertainties, decision making process of people can be unexpected and complicated due to dependent factors and dynamic environment. However, prediction and analysis of human decision making process is fundamental in sociology and other domains where the analysis of human behavior is required. For instance, in market management area, market analysis, studies on consumer decision making and other means for finding the most optimal marketing solution are vital but easily ignored steps for small merchants and individual businesses. This is especially salient during COVID-19 pandemic period, in economic domains, due to the great changes in international trade, transportation and relationships. Similar events are frequent during the COVID-19 pandemic in 2020. Government and social organizations tend to implement either non-action or extreme action on making rules of travel, rarely finding efficacious solution on human behavior, leading to conflicts and unnecessary casualties.

According to the specified situation, a theory and related model is needed in this research to guide and qualify the research. Among those effective theories proposed in earlier period, DFT was proposed as a dynamic cognitive solution to model human decision making process, and has been continuously modified to improve its performance in more complex environment. The model aims at dynamically cognizing people's behavior through the known data collected in the past, and giving prediction and rational explanation of the future trend of behavior. DFT considers two significant factors rarely seen in other theories, which are variability of preference of choices, and systematic relation between preferences and deliberation time [3]. The two factors endow DFT model flexibility in time scale and higher accuracy of simulation in the decision making process. DFT is distinguished from the previous approaches and successfully applied in various domains. It is not only a suited model for this study case, but also providing good performance to motivate the research work.

Human decision making process are classified into three categories: economical decision making, psychological decision making, and synthetic engineering-based decision making [4]. Compared with economical decision making approaches, psychological approaches can have better performance on representing human cognitive natures (e.g., memory, and mood) which are of psychology. DFT is a theory and mathematical model in psychology field based on psychological principles [5].

Shao extended DFT with Analytical Hierarchy Process (AHP) to improve its performance in complex dynamic environment of decision making, and proved it is capable of predicting people's behavior in class attendance context [2]. In reality, human decision making process during pandemic can greatly vary from the previous because any event or new outbreak is possible to happen and affects the current preference evaluation of people, which is regarded as a dynamic process.

In DFT theory, the evaluation value of attribute and attribute number are dynamic under complex environment. Movement decision making is such a dynamic case, especially during the worldwide COVID-19 pandemic. People would consider at least the two attributes (1) the risk of going out and (2) the desire of going out in their decision making on trip options. The evaluation of attributes is obviously dynamic since the situation of pandemic is varying all the time, even with dramatic changes. This is a complex environment under uncertainty. Moreover, people may take other attribute into consideration, for instance, the prevention or orders from society, as the pandemic develops.

It is proved that DFT is a strong model to explain the phenomenon of human behavior. It is particularly suited for the complex context in this research. To reflect the character of dynamic environment, this study is conducted under the guideline of the extended DFT model to include the situation of people going out during the COVID-19 pandemic in 2020. This context 
is in daily life and expected to further verify the performance of extended DFT model in dynamic environment with quantifying the collected data with model.

\section{DECISION FIELD THEORY}

DFT is a dynamic-cognitive approach applied in understanding the cognitive mechanism of the deliberation process in human decision making under uncertainty. It was initially proposed to be a deterministic-dynamic model of approach-avoidance conflict behavior [6]. Subsequently, it was updated as a stochastic-dynamic model for decision making process.

\subsection{Development of DFT}

DFT has been changed and modified for a few times, which laid the foundation of the current theory and model. Deterministic Subjective Expected Utility (SEU) theory was initially proposed following the laws of probability theory, whereas the number of choices is restricted to be two [7]. The Deterministic SEU Theory is able to explain the trend of preference on options, but conflicts with the dynamic change of preferences. Deterministic SEU is able to explain only the trend of preferences on options, but bot capable to understand the dynamic change of preference states. This weakness has been solved by the Sequential SEU Theory proposed in 1970 [8]. In this theory, the preference switches on relevant factors sequentially and regularly in the period. However, the theory cannot take the factors in the past into consideration, which means the experience and knowledge in the past cannot affect the current decision-making process, and the initial preference state was always set to be zero. This problem of the theory produces imprecision in explaining really human decision-making process in psychology. The Random Walk SEU Theory added an initial state into the previous model and solved this weakness [9]. The deterministic decision making theories primarily originated from binary preference relation [10]. Subsequently, it was adapted to support multi-attribute decision making under dynamic environment [11], and generalized to accommodate multiple options [5], which make it available for modelling decision making based on various options. Whereafter, the decision-making theories were developed to current stage.

DFT has been applied across a broad range of domains such as sociology, psychology, and supply chains [12]. In stock market domain, researchers built a DFT-based model to estimate the preference states under uncertain environment, where the current risks are relevant to past experiences [13]. In supply chain management area, Sucky proposed a dynamic decision making approach to do strategic vendor selection or switching based on hierarchical planning principles [14]. It was soon proved that the approach has a good performance in explaining decision making in vendor selection and giving optimal selection in vendor management. More than these, DFT is also applied in many other areas and tasks, e.g., disaster management [15], preferential choice [16], and urban water management [17], etc.

In a psychological decision-making process, attribute and weight value are two significant components. Attributes are generally various influential factors having impacts in decision making. Accordingly, higher value of a particular attribute makes decision maker tend to the particular one option or options, which can also be called preference. The weight value is the attention that decision maker allocated to each attribute [18], it can be seen as priority of each attribute. A very common instance is that people would generally consider price and quality as two factors, i.e., attributes in purchase decision making between car A and car B. The number of car price and value of performance are evaluation values of two attributes. The weight value is how important each attribute is to the decision maker in purchasing a car. If the buyer is more price-sensitive, the weight value of attribute price will be assigned higher than the attribute quality. In the DFT model, only the weight value is changeable in deliberation process while the attributes and evaluation value are fixed. The DFT model performs well in this case, since the values of price and performance, i.e., evaluation values of two attributes are relatively stable. While the attention the buyer assigned on attributes will change as buyer's cognition to the car varies.

\subsection{DFT Model}

As mentioned in the proposed theories, DFT was originally introduces in 1993 to model a dynamic cognitive approach for decision making process [3], which has been applied in various areas. Among the variants of DFT, the latest original model work with multiple options and attributes was proposed in 2001 [5]. The main idea of DFT is that the human decisionmaking process focuses on two main factors: experiences and current evaluations. In mathematical format, the dynamic evolution of preference is expressed as Eq. (1).

$$
P(t+h)=S P(t)+V(t+h)
$$


In the Eq. (1), $P(t)^{T}=\left[P_{1}(t), P_{2}(t), P_{3}(t), P_{4}(t)\right]$ represents the vector of preference values where $P_{i}(t)$ indicates the strength of preference corresponding to option $i$ at time $t$. Time $t$ and $t+h$ are adjacent time points. The parameter $h$ is a time unit representing the increment between the two adjacent time moments in decision making process. The time $h$ has the limit of zero and can be an arbitrary value greater or equal to zero.

$S$ is a symmetric matrix called growth-decay matrix or feedback matrix in which the diagonal elements provide forgetting factors which represents the memory of the previous values in the model, and the non-diagonal elements provide competitive interactions among alternatives [19]. Current information and knowledge is often not the only factor that determines the cognition of decision maker, memory and previous knowledge also matter in the process of making a decision [20].

$V(t)$ indicates the valence vector for all options at time $t$, which is composed of three matrices as Eq. (2).

$$
V(t)=C M W(t)
$$

Attribute matrix $M$ is an $n \times m$ matrix in which $n$ refers to the number of options and $m$ refers to the number of attributes. The matrix $M$ indicates personal preferences on options. The attribute matrix $M$ includes the evaluation values for each option on the attribute in various situations. For instance, people would consider their desire of going out and the prevention from family as two different attributes when they make the decision to go out or not. The two examples of attributes happen to be oppositely affecting the decision. This is how people feel difficult to make a decision in daily life.

Matrix $W$ is an $m \times 1$ matrix called weight matrix, in which $m$ is the number of attributes. It represents the importance of different attributes in $M$. People allocate different weight values in matrix $W$ to indicate personal evaluations of each attribute.

Matrix $C$ is called contrast matrix in which all the options will be compared with each other. The construction of this $n$ dimensional matrix guarantees the increase of preference value to one option will lead to the decrease of preference value to the other, which means the summation of all preference values fixedly equals to zero.

The process of making a decision is the process in which people's preference state changes among various options. The changes are determined by dynamic valence, which is consist of three components: contrast matrix $C$, attribute matrix $M$, and wight matrix $W(t)$. The value of contrast matrix $C$, and attribute matrix $M$, is fixed while the value of $W(t)$ is changeable. In the human decision making process, weight values of different attributes change from one to another while new information entering this environment, leading to the changes of valence value and preference states. This is a dynamic changing process.

In this original DFT model, the attribute matrix $M$ is dedicated as a static parameter. Nevertheless, in reality, the environment is more complex and dynamic, and the attributes cannot be defined only at the first [21]. More importantly, the value of evaluation on each attribute are also dynamic along with people understanding the problem better.

Aiming to solve this problem, the original DFT model was extended by integrating with the structure of Analytic Hierarchy Process [2]. The extended model is capable to add or delete attributes, and values of attributes are changeable. Compared with the original DFT equation, the structure of contrast matrix $C$ and feedback matrix $S$ are same, and the parameter of attributes matrix $M$ and weigh matrix $W$ changed from $t$ to $t+h$. In original DFT model, the summation of all weight values of attributes are in random number. In order to standardize the DFT model, the sum of weight values is set always to be equal to 1 which learnt from dynamic AHP structure as well.

\section{METHODOLOGY}

It is urgent to know if the extended DFT model is able to explain and predict people's behavior during COVID-19 pandemic, then make more effective strategies tackling the spreading of pandemic. According to that purpose, the methodology of this research was developed, and driven by DFT model. The data collection was designed according to the structure and elements of DFT model. At the end, the descriptive results of data analysis are presented.

\subsection{Data Collection}

Data of people's movement decisions is required in the research process of using DFT model to quantify research questions and describe the phenomenon. Thus, data collection was conducted in early stage. The data collecting method was web survey containing three rounds of survey, with an average time interval in 10 weeks. By means of an online questionnaire service, the efficiency and accuracy of data collection, and privacy of participants are guaranteed. The online survey platform Tencent Survey (https://wj.qq.com/) was used to organize web survey, and distributed 
questionnaires by sending QR Code for survey web to participants respectively.

\subsection{Background of Data Collecting}

The data collection was conducted in China mainland. People who participated in the collecting process are all from Jiangsu and Shandong province. In the duration of data collection, the situation of COVID-19 pandemic, and the guidelines and restrictions from the government had changes, which can be seen as newly entered information in a dynamic environment under uncertainty. The round 1 started half a month after the beginning of the new semester. This is the time when the participants were familiar to the new movement rules, and formed new movement habits after returned to school. The second round of survey started in the middle of a holiday week for China's National Day and Mid-Autumn Festival. In the holiday, people's trip decisions were strongly affected by new factors and changes of environment because of a big population mobility caused by traditional customs in Mid-Autumn Festival [22]. Returning home and having family reunion in Mid-Autumn Festival is unchangeable in many people's minds [23], people's travelling restrictions in society were thus changed as they entered to a new place and environment. In the period of survey round 3, the weather turned to be colder and new factors affecting people's trip decisions occurred. The whole process of data collection has included different factors changed in respects of society, government, customs, and climate. The factors were from both internal and external.

\subsection{Attributes in DFT Model}

In DFT model, attribute matrix indicates the evaluation for each option on different attributes. Various reasons for particular behavior can be seen as the relevant influential factors that affect those behaviors of people independently of each other. In this study, the movement decision making environment is the environment in which the evaluation value of attribute and attribute value will dynamically change, and the decision making problem is the problem in such environment. In our scenario, the dynamic problem of movement rules will be analysed in terms of several aspects.

For a simple and effective model, small interviews were previously conducted. The main aim of the interviews was to understand why people would decide to go out or not during the pandemic. The top four reasons for going out or not were confirmed to be the factors that affect people's decision making process on movement during pandemic. There are two factors for not going out are Prevention by government/society/family and Fear of COVID-19. For the going out decision, the attributes are termed as Desire of going out and Invitation from friends. The number and values of attributes evaluation are dynamic as time changes. Thus, the four attributes are not constant. The fifth attribute in addition to those selected attributes will be added into attribute matrix according to the results of web survey.

The movements options during pandemic are initially defined as the four methods: trip inside local city, trip outside local city, trips in both ranges of inside and outside city, and neither of the trips. Based on the attributes and people's possible movements options discussed above, the attributes are defined in Table 1.

Table 1. Pre-defined attributes

\begin{tabular}{|l|l|}
\hline No & Attributes \\
\hline A1 & Prevention by governments/society/family \\
\hline A2 & Fear for COVID-19 \\
\hline A3 & Desire of going out \\
\hline A4 & Invitation from friends \\
\hline
\end{tabular}

\subsection{Design of Web Survey}

The purpose of this survey is to study the dynamic process of decision making of people for options of going out or not during pandemic regarding specified aspects. There were three separate rounds of web survey, the data collected from each round were analysed in order to quantify extended DFT model and learn mobility rules of people during COVID-19 pandemic.

\subsubsection{Arrangement of Survey}

The three rounds of survey were carried out based on the mobility decision making process of people during pandemic, and the participants were able to freely leave home or where they live and go to available public places. There were 52 people enrolled in the survey, and they voluntarily participated each round of survey.

The first round of survey begun from September 17, 2020, and the third round ended on November 24, 2020. In each round there were different factors affecting people's decision making on moving. The duration of all web surveys was about 10 weeks which was divided into three periods. Time duration of each round of survey is presented in Table 2 . 
Table 2. Time duration of each round of survey

\begin{tabular}{|l|l|l|l|}
\hline Round & Start date & End date & Duration \\
\hline Round 1 & Sept 17 & Sept 21 & 5 days \\
\hline Round 2 & Oct 7 & Oct 19 & 13 days \\
\hline Round 3 & Nov 9 & Nov 24 & 16 days \\
\hline
\end{tabular}

All guidelines and instructions were given to all participants at the beginning of round 1 . The period of round 1 was in normal weeks and lasted for 5 days, which is better than our expectation. There was an interval of 17 days between round 1 and 2. The beginning date of round 2 was still in a holiday week. The round 3 started in Nov 9 and lasted 16 days long. The time interval between the latter two rounds was 20 days. After two rounds of survey, people were less motivated in survey 3 . Thus, it took the longest time to collect answers from people.

\subsubsection{Question Statements}

The answers to the questions were used in applying the extended DFT model and conclude information and rules behind movements behavior of people. The questions were not exactly the same in each round, since the elements are dynamic in this model.

In the first round of survey, there were six questions used to quantify the extended model, and one question to acquire the additional attribute. For the questions 2 to 5 the grading scale was set from 1 to 7 to evaluate the extent of each option. For question 7 , the scale was set from 1 to 5. A greater number indicated a higher agreement on the related statement of the question. Moreover, the question 6 is used to acquire one additional attribute. A brief description of questions is as follows.

The first question was designed to obtain the intended behavior of participants on movement decision making. The answer to question 2 is used to value the elements of the attribute Prevention by government/society/family on different options. Similarly, questions from question 3 to question 5 aim at evaluating decision-making of the attributes Fear for COVID-19, Desire of going out, and Invitation from friends, respectively. The answer to question 6 is the key question to change the numbers of attributes in the extended model. It was assumed that there are new attributes as time goes or condition changes, providing additional attributes as options. The attribute selected the most by participants will be the fifth attribute in the extended DFT model. The answer to question 7 is used to value the weight values of each attribute. The additional attribute is also included in the latter two rounds.
In round 2 and 3 , the fifth attribute was added according to the answers to question 6 from participants. Therefore, the survey in round 3 also had an additional question which was set to get the evaluation values of the fifth attribute for four options. Hence, the number of attributes listed in question 7 increased from 4 to 5 in round 2 and 3.

\section{RESULTS}

After data collection through three rounds of web survey and preliminary data analysis, the results are visualized and represented in this part. The original data was collected based on four options, which are respectively Trip inside local city, Trip outside local city, Trip in both ranges, and Neither of the trips. However, only a very small number of participants decided to have trips outside the city in both round 1 and round 3. To make the analysis more logical and intuitively correct, data on the option Trip outside local city was merged into the option Trips in both ranges due to some defects of this option. Any movement that related to the outside of city will be regarded as the option Trip in both ranges. The data is presented in Figure 1.

\subsection{Participation Rate}

The number of participants and the participation rate in each round of survey are presented as follows. We contacted 52 people to voluntarily participate in the three rounds of survey before the first round. There were 51 participants finished the first round of survey. The main range of ages of the participants in round 1 is from 18 to 50 , and only three participants are older than 50 years. There are 27 participants studying at school, and 18 of participants have full-time jobs. The participation rate decreased to $81 \%$ in round 2 with their motivation drops. In round 3, there were 39 participants finished the web survey, the participation rate fell to $75 \%$. The data is presented in Table 3.

Table 3. Participation rate in each round of survey

\begin{tabular}{|l|l|l|}
\hline Round & $\begin{array}{l}\text { Number of } \\
\text { participants }\end{array}$ & $\begin{array}{l}\text { Participation } \\
\text { rate }\end{array}$ \\
\hline Round 1 & 51 & $98 \%$ \\
\hline Round 2 & 42 & $82 \%$ \\
\hline Round 3 & 39 & $75 \%$ \\
\hline
\end{tabular}

\subsection{Data Visualization}

The data was collected from seven basic questions in round 1 , plus one additional question in round 2 and 3. The visualized data of these questions are as below. 
After collecting and sorting the answers to question 1 in the three rounds of survey, the intended behavior of people for three options of movement decisions is depicted in the Figure 1.

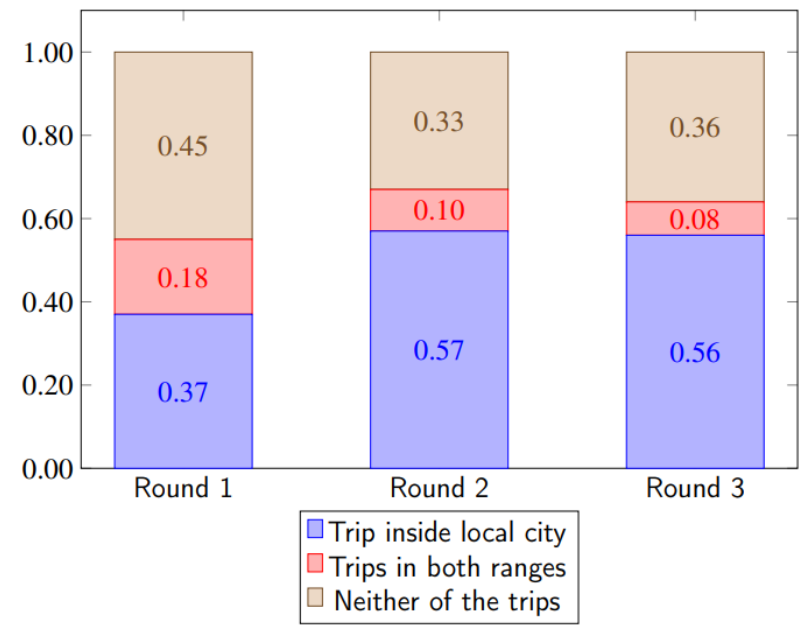

Figure 1 Intended behavior of people on movement decision making.

Seeing from the bar chart in Figure 1, it is obvious that most people were willing to have small trips inside the city range, the behavior is especially distinguished in round 2 and round 3. Meanwhile, the proportion of participants who prefer to stay at home rather than any kind of movements reached the peak in round 1 . Between the latter two rounds of survey, a great similarity is presented. In round 2 , the number of people who want to go out has increased from round 1 , and within which the local trip value reached the highest point among the three rounds. Meanwhile, fewer people would like to stay at home. The number of people who want to go outside the city (including the options Trip outside local city and Trips in both ranges) generally fell in the latter two rounds. The data of round 3 is not distinguished from the round 2 . However, there are still small changes that can imply behavior trend or rules behind it. The number of participants who travel inside the city very slightly decreased from round 2 and cannot be regarded as a change. The proportion of people who have trips in both ranges is narrower. It is visible that more people decide to stay at home.

Considering the data with its corresponding date in which particular events happened, rational deduction and reasoning can be given as follows. The first round of survey started at September 27 and lasted for 5 days. For most people, especially students, they have stayed at home for more than eight months due to COVID19 , and it requires time to accommodate new environment and lifestyle when the environment changed to school from home. A majority of our participants are students who are studying in universities or colleges, and that period of time for survey round 1 located in the latter half of the first month that students went back to school from home, which left a cushion for people. As expected, being affected by the Stay-At-Home order, the majority still tend to stay indoors, and the proportion is 0.45 , exceeded all other rounds. That is to say, $45 \%$ of all participants chose to have city-ranged movements over other options in round 1. Correspondingly, the percentage of participants who chose to have cityranged travels reached the lowest point among three rounds at 0.37 . Nevertheless, the proportion of Trips in both ranges (including those people whose go outside of local city no matter if they still have travels inside city range) in round 1 is also larger than the other two rounds, which appears to be unreasonable. A convincing explanation can be that students tend to have travel at a greater sense to satisfy the desire of going out, especially after coming back to school with looser Stay-At-Home order than at home. This situation created possibility and even opportunity to have travels outside city. Consequently, the whole process of how it has changed is demonstrated as data of round 1 in Figure 1.

The second round of survey started at October 7 and lasted for 13 days. Data in round 2 depicts a different tendency from round 1 , even opposite. The proportion of people who have trips inside local city peaked among three rounds at 0.57 , while only 33 percent of participants wanted to stay at home. People obviously showed interests in city-ranged travels. The reason is possible to be related to the date. October 7 is in a holiday in which those who work or study in other cities may return hometown, as discussed in Chapter 4.2. The "local city" for participants was possibly changed during that time. Consequently, various factors are dynamic, and the environment is complex in the context. In one's hometown, movement behavior can be greatly influenced by some particular factors, e.g., invitation from old friends or reunion with family and relatives can play an important role in this data. Due to the same reason, less people travelled to other cities.

In survey round 3 , it can be seen form data that more people tend to stay indoors, and less people had travels inside or outside the city notwithstanding the exiguity of the data changes. This is possibly due to the weather change when the data was being collected. Moreover, the time duration of survey round 3 was 16 days long. Approaching winter, the weather changes particularly fast in the season. For COVID-19, a temperature-sensitive virus, weather and temperature 
can greatly change pandemic situation and then lead to different human behavior and travel pattern. Accordingly, the temperature and weather changes can be the reason for data changes in round3.

According to the data collected through question 7 , the weight values of attributes in three rounds are demonstrated as following Figure 2.

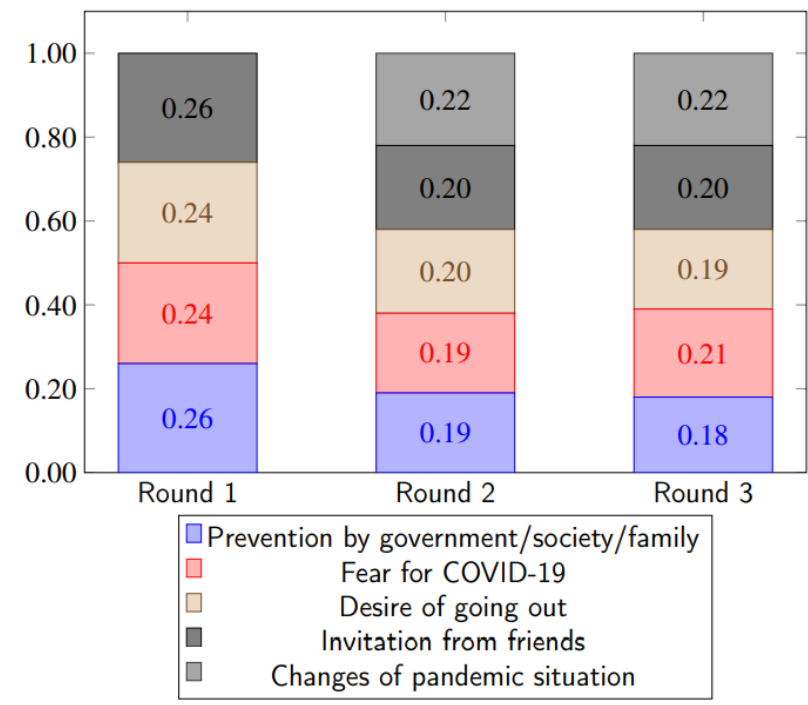

Figure 2 Weight value of attributes in three rounds

As discussed above, weight value is one of the two significant components in human decision making process, which represents the attention that decision maker allocated the attribute. It can also be seen as the importance of each attribute to decision maker. It clearly indicates that none of these attributes distinguishes greatly from others. However, the tiny differences can lead to diverse results when it is used to quantify DFT model.

A feature of the extended DFT model can also be observed from this figure. Due to the extension to original DFT model, the performance and flexibility is improved for dynamic changes, as then the additional attribute joined, there is data for 4 attributes in round 1 , while 5 attributes in round 2 and round 3 . This reflects the dynamic change when people make travel decision in such complex and dynamically changing environment, including the process of new factors entering consideration as pandemic develops.

\section{CONCLUSIONS}

In COVID-19 pandemic, efficient management solutions and strategies are demanded to fight against the pandemic, in which research in human behavior field, especially those behavior on movement decisions is of great significance. Decision Field Theory is a reliable model in human behavior and decision making fields with high performance. This paper discusses the process of human decision making on movements during COVID-19 pandemic. Data collection of this research was conducted according to the structure and elements of an extended DFT.

Descriptive results and reasoning of data analysis is given in this paper. It is found that as the situation of pandemic changes, human behavior of movements is also affected. As time changes, there are also other factors enter into consideration and affect decision making. For example, the fifth attribute was dynamically added as additional attribute in round 2 and 3 by participants. At different time, the importance of different factors varies in deliberation. In our research context, survey round 2 was in a holiday with traditional festival in which people meet family and old friends. At that time, the attribute Invitation from friends may had greater attention, meaning it affects people's decision to a greater degree than other factors. In the later survey round 3, weather and temperature changes possibly had a bigger impact on people's decision making on movements, by affecting pandemic situation.

There are also potential improvements in this paper. For instance, due to the location and time of data collection, the differences among three rounds indicated in data were not very distinguishable. The data was collected in China when the COVID-19 pandemic was relatively stable and dampened. The data can show the changes better if it could have been conducted in other places outside China, and different. Moreover, the changes would be more dynamic if survey intervals between rounds can be longer, the results could also be more accurate by doing so. Further study could analyse the collected data deeper, by quantifying with the extended DFT model. The results can also be in a higher level and present more details by fully applying the model in mathematical way. The performance of extended DFT could be test by this research with the data. 


\section{REFERENCES}

[1] J. T. Cacioppo and L. C. Hawkley, 'Perceived social isolation and cognition', Trends Cogn. Sci., vol. 13, no. 10, pp. 447-454, Oct. 2009, doi: 10.1016/j.tics.2009.06.005.

[2] L. Shao and J. Markkula, 'Decision Filed Theory', in Encyclopedia of Information Science and Technology, Fourth Edi., vol. 43, no. 01, M. Khosrow-pour, Ed. IGI Global, 2018, pp. 43-0002-43-0002. doi: 10.5860/choice.430002 .

[3] J. R. Busemeyer and J. T. Townsend, 'Decision field theory: A dynamic-cognitive approach to decision making in an uncertain environment.', Psychol. Rev., vol. 100, no. 3, pp. 432-459, 1993, doi: 10.1037/0033-295X.100.3.432.

[4] S. Lee, Y.-J. Son, and J. Jin, 'Decision field theory extensions for behavior modeling in dynamic environment using Bayesian belief network', Inf. Sci., p. 18, 2008.

[5] R. M. Roe, J. R. Busemeyer, and J. T. Townsend, 'Multialternative decision field theory: A dynamic connectionist model of decision making', Psychol. Rev., vol. 108, no. 2, pp. 370-392, 2001, doi: 10.1037/0033295X.108.2.370.

[6] Townsend, James T and Busemeyer, Jerome R, 'Approach-avoidance: return to dynamic decision behavior', in Current Issues in Cognitive Processes: Tulane Flowerree Symposium On Cognition, 1st ed., Chizuko Izawa, Ed. Psychology Press, 1989, pp. 107133.

[7] L. J. Savage, The foundations of statistics. New York: Wiley, 1954. doi: https://doi.org/10.1002/nav.3800010316.

[8] Morris H. DeGroot, Optimal statistical decisions. Wiley-Interscience, 1970. doi: 10.2307/2987329.

[9] J. R. Busemeyer, 'Decision making under uncertainty: A comparison of simple scalability, fixed-sample, and sequential-sampling models.', J. Exp. Psychol. Learn. Mem. Cogn., vol. 11, no. 3, pp. 538-564, 1985, doi: 10.1037//0278-7393.11.3.538.

[10] P. C. Fishburn, 'Expected utility: An anniversary and a new era', J. Risk Uncertain., vol. 1, no. 3, pp. 267-283, Sep. 1988, doi: 10.1007/BF00056138.

[11] A. Diederich, 'Dynamic stochastic models for decision making under time constraints', $J$. Math. Psychol., vol. 41, no. 3, pp. 260-274, 1997, doi: 10.1006/jmps.1997.1167.

[12] J. R. Busemeyer and A. Diederich, 'Survey of Decision Field Theory’, Math. Soc. Sci., vol. 43, pp. 345-370, 2002.
[13] M. Cohen, J. Etner, and M. Jeleva, 'Dynamic decision making when risk perception depends on past experience', Theory Decis., vol. 64, no. 2-3, pp. 173-192, 2008, doi: 10.1007/s11238007-9061-3.

[14] E. Sucky, 'A model for dynamic strategic vendor selection', Comput. Oper. Res., vol. 34, no. 12, pp. 3638-3651, 2007, doi: 10.1016/j.cor.2006.01.006.

[15] V. Vescoukis and N. D. Dulamis, 'Disaster management evaluation and recommendation', Proc. - 20113 rd Int. Conf. Games Virtual Worlds Serious Appl. VS-Games 2011, pp. 244249, 2011, doi: 10.1109/VS-GAMES.2011.43.

[16] K. M. Aschenbrenner, D. Albert, and F. Schmalhofer, 'Stochastic choice heuristics', Acta Psychol. (Amst.), vol. 56, no. 1-3, pp. 153166, Aug. 1984, doi: 10.1016/00016918(84)90015-5.

[17] L. J. Pearson, A. Coggan, W. Proctor, and T. F. Smith, 'A Sustainable Decision Support Framework for Urban Water Management', Water Resour. Manag., vol. 24, no. 2, pp. 363376, Jan. 2010, doi: 10.1007/s11269-009-94501.

[18] Lan Shao, 'An Extended Model of Decision Field Theory Integrated with AHP Structure for Complex Decision Making Problems', 2015.

[19] S. Lee and Y. J. Son, 'Extended decision field theory with social-learning for long-term decision-making processes in social networks', Inf. Sci., vol. 512, pp. 1293-1307, 2020, doi: 10.1016/j.ins.2019.10.025.

[20] James C. Van Horne \& George G.C. Parker, 'The Random-Walk Theory: An Empirical Test', no. December, 1967.

[21] T. L. Saaty, 'A scaling method for priorities in hierarchical structures', J. Math. Psychol., vol. 15, no. 3, pp. 234-281, Jun. 1977, doi: 10.1016/0022-2496(77)90033-5.

[22] J. Pan and J. Lai, 'Spatial pattern of population mobility among cities in China: Case study of the National Day plus Mid-Autumn Festival based on Tencent migration data', Cities, vol. 94, no. 967, pp. 55-69, 2019, doi: 10.1016/j.cities.2019.05.022.

[23] J. J. V. Bavel et al., 'Using social and behavioral science to support COVID-19 pandemic response', Nat. Hum. Behav., vol. 4, no. 5, pp. 460-471, 2020, doi: 10.1038/s41562-020-0884$\mathrm{z}$. 